\title{
Cinza de palha de cana-de-açúcar como adição mineral em fibrocimento
}

\author{
Michelle S. Rodrigues ${ }^{1}$, Antonio L. Beraldo ${ }^{1}$, Holmer Savastano Júnior ${ }^{2}$ \& Sergio F. Santos ${ }^{2}$
}

\section{RESUMO}

O Brasil é o maior produtor mundial de cana-de-açúcar e sua indústria sucroalcooleira gera grande quantidade de resíduos líquidos e sólidos, dentre eles a palha da cana-de-açúcar. Com o objetivo de agregar valor a este resíduo produziu-se, neste trabalho, cinza de palha de cana-de-açúcar por meio da queima controlada a $700{ }^{\circ} \mathrm{C}$ e se avaliou sua aplicação como adição mineral em compósitos de fibrocimento. A cinza foi caracterizada pelas técnicas de fluorescência de raios $X$, difração de raios $X$, granulometria a laser e superfície específica. Nos compósitos foram avaliadas as propriedades de absorção de água, porosidade aparente, massa específica aparente, módulo de ruptura, energia específica, módulo de elasticidade e limite de proporcionalidade. A caracterização das cinzas indicou superfície específica e porosidade elevadas, além de apresentar um halo de amorficidade no seu difratograma. Compósitos produzidos com as cinzas mostraram características físicas e mecânicas similares àquelas do controle o que viabiliza a substituição parcial do cimento Portland por esse tipo de cinzas.

Palavras-chave: pozolana, resíduos agroindustriais, porosidade

\section{Sugarcane straw ash as mineral addition in fiber-cement}

\begin{abstract}
Brazil is the world's largest producer of sugarcane, and its sugarcane industry generates a large amount of liquid and solid wastes, among them the sugarcane straw. Aiming to add value to this waste, sugarcane straw ash was produced by controlled burning at $700{ }^{\circ} \mathrm{C}$ and it was applied as mineral addition in cement composites. The ashes were characterized by the techniques of $X$ ray fluorescence, $X$ ray diffraction, laser particle size and specific surface area. The following composite properties were evaluated: water absorption, apparent porosity, bulk density, modulus of rupture, specific energy, elastic modulus and proportionality limit. The characterization of ash showed a high specific surface area and porosity and a halo of amorphicity was observed in the diffractograms. Composites produced with these ashes showed physical and mechanical characteristics similar to that of the control, allowing the partial replacement of Portland cement by this type of ashes.
\end{abstract}

Key words: pozzolan, agro industrial wastes, porosity 


\section{INTRODUÇÃO}

Com a expansão da atividade da construção civil no Brasil, o consumo per capita de cimento Portland foi, em 2010, de 311 $\mathrm{kg}$ por habitante e representou um aumento de $15 \%$ em relação ao consumo de 2009. A indústria brasileira de cimento possui, atualmente, um parque industrial moderno e é reconhecida internacionalmente por apresentar baixas emissões de $\mathrm{CO}_{2}$ e pelo uso de adições minerais e de biomassa na geração de energia (SNIC, 2012).

$\mathrm{O}$ uso de cinzas volantes, de sílica ativa, metacaulim e de outros materiais pozolânicos permite reduzir substancialmente o consumo de cimento Portland (Mitchell et al., 1998; Payá et al., 2001; Isaia et al., 2003; Mishra et al., 2003; Frías \& Rojas, 2005). Desta forma, a utilização de resíduos adequados em uma matriz cimentícia pode contribuir para que a indústria de materiais cimentícios se torne mais sustentável (Dwivedi et al., 2006).

Muitas pesquisas sobre as aplicações de resíduos agroindustriais na forma de adições minerais têm-se desenvolvido nas últimas décadas, dentre as quais se destacam os estudos efetuados com as cinzas de casca de arroz (Bui et al., 2005; Tashima et al., 2011; Sata et al., 2012) e as cinzas de bagaço e de palha de cana-de-açúcar (Martirena et al., 2006; Ganesan et al., 2007; Frías et al., 2011; Guzmán et al., 2011), que se situam entre os resíduos gerados em quantidades significativas na agroindústria brasileira.

Estudos com novos compósitos de fibrocimento vêm-se intensificado nos últimos anos. Pesquisadores e fabricantes têm buscado a utilização de fibras alternativas ao amianto (Tonoli et al., 2011). O uso de fibras vegetais pode mostrar-se como opção viável por se tratar de um material renovável e de baixo custo, quando comparadas às fibras sintéticas.

Na preparação de compósitos à base de cimento Portland e fibras vegetais devem ser considerados dois fatores - o ataque alcalino às fibras e a incompatibilidade física e química entre as fibras e a matriz. A degradação de materiais cimentícios modificados pela adição de fibras vegetais ocorre principalmente em virtude da elevada alcalinidade da água presente nos poros da matriz do cimento Portland (com pH superior a 13). A interface, região da matriz em torno da fibra, tem alta porosidade e permite o acúmulo de água; com a presença de quantidades elevadas de $\mathrm{Ca}(\mathrm{OH})_{2}$, propicia-se a formação de uma região com elevada alcalinidade (Agopyan \& Savastano Jr., 2003).

Esses materiais reforçados com fibras vegetais podem ter seu desempenho mecânico comprometido pelo alto nível de alcalinidade, visto que o ataque do meio alcalino às fibras provoca sua degradação causando perda de resistência mecânica do compósito (Peruzzi, 2007).

Uma solução para minimizar esse ataque exercido pela matriz é a substituição parcial do cimento por pozolanaso que pode diminuir a alcalinidade da matriz em consequência do consumo de hidróxido de cálcio pela reação pozolânica e/ou pela menor quantidade de clínquer presente (Nita, 2006).

Em estudos desenvolvidos com o uso de pozolanas a fim de se reduzir a alcalinidade da matriz, pôde-se observar grandes melhorias obtidas com a utilização de cimento com elevado teor de alumina e de pozolanas naturais; a substituição de $45 \%$ de cimento por sílica ativa manteve a tenacidade do compósito. No entanto, com a substituição parcial do cimento Portland por cinza volante ou escória, nenhuma melhoria significativa foi obtida (Toledo Filho et al., 2003).

Lima \& Toledo Filho (2008) estudaram matrizes cimentícias com $30 \%$ de metacaulim (MK), com o intuito de melhorar o desempenho de compósitos reforçados com fibras de sisal. Os autores observaram que com a utilização desse teor de MK tornou-se possível produzir uma matriz livre de cal $(\mathrm{CH})$ e que tenha apresentado resistência mecânica mais elevada, menores absorção e índice de vazios, se comparada à matriz controle. Nas matrizes livres de $\mathrm{CH}$ os autores constataram que as fibras mantiveram o comprimento de arrancamento e não apresentaram alterações visíveis após o ensaio de envelhecimento acelerado.

Dentro deste contexto estudou-se, neste trabalho, a aplicação de cinza de palha de cana-de-açúcar (CPCA) obtida mediante condições de queima controlada em compósitos de fibrocimento, com o intuito de melhorar as características mecânicas da matriz.

\section{Material e Métodos}

Com vista à preparação dos compósitos de fibrocimento foram utilizados os seguintes materiais: cimento Portland de alta resistência inicial (CPV-ARI), da marca Cauê (produzido na cidade de Ijaci, MG), a pureza e a granulometria do cimento Portland comercial justificam seu uso neste trabalho uma vez que o mesmo não contém adições minerais, apresentando maiores quantidades silicatos tricálcico e dicálcico, o que faz com que sua reatividade seja aumentada; polpa celulósica branqueada de eucalipto (Eucalyptus urograndis), com o intuito de melhorar a distribuição do reforço dentro do compósito e para auxiliar a retenção de partículas durante a sucção na moldagem adotada neste trabalho; fibras de bagaço de cana-de-açúcar e cinzas de palha de cana-de-açúcar (CPCA) produzidas mediante queima controlada a $700{ }^{\circ} \mathrm{C}$.

\section{Tratamento das fibras de bagaço de cana-de-açúcar}

As fibras foram coletadas na usina Abengoa Energia, na cidade de Pirassununga, SP, e após o recebimento passaram por um processo de lavagem em água quente a $100^{\circ} \mathrm{C}$, durante 30 min, com o objetivo de eliminar as impurezas que poderiam inibir as reações de hidratação do cimento Portland. As fibras utilizadas nos compósitos foram aquelas que passaram pela peneira de abertura de 2,38 mm (\# mesh 8) e ficaram retidas na peneira de abertura de 1,19 mm (\# mesh 16).

\section{Produção da CPCA}

A palha utilizada na produção das cinzas foi coletada no Centro de Tecnologia Canavieira (CTC) em Piracicaba, SP. $\mathrm{O}$ material foi colocado em bandejas para secagem natural (nos meses de agosto, setembro e outubro de 2011, na cidade de Pirassununga, com temperatura média de $21,9^{\circ} \mathrm{C}$ além de umidade relativa média de $42 \%$ ) e posteriormente levado à queima em um forno mufla, da marca Jung, modelo 10013, com dimensões internas de $27 \times 40 \times 100 \mathrm{~cm}$. A queima das cinzas 
foi realizada em dois patamares a fim de ser o mais homogênea possível: primeiro, a queima foi realizada a $400{ }^{\circ} \mathrm{C}$ durante 20 min e em seguida realizada a $700{ }^{\circ} \mathrm{C}$ durante $60 \mathrm{~min}$. A taxa de aquecimento foi de $10{ }^{\circ} \mathrm{C} \min ^{-1}$ e o resfriamento das cinzas foi lento; após a queima as cinzas foram levadas a um moinho de bolas da marca Tecnal, com cerca de 70 bolas e com a massa correspondente a $3000 \mathrm{~g}$, no qual foi conduzida a moagem durante 120 min, adotando-se a velocidade de rotação de 200 RPM.

\section{Caracterização física e química da CPCA}

A caracterização da CPCA foi realizada por meio das seguintes análises: fluorescência de raios $\mathrm{X}$ (para obter a composição química do material, em um espectrômetro por fluorescência de raios X Axios, marca Pan analytical), difração de raios $\mathrm{X}$ (em um difratômetro de 6 círculos Huber, com cristal analisador $\mathrm{Ge}(111)$ e detector linear Mythens a $9 \mathrm{keV}$ $(\lambda=1.3776)$ de alta resolução, do Laboratório Nacional de Luz Sincrotron), distribuição granulométrica a laser (analisador de partículas Malvern Mastersizer 2000) e superfície específica pela técnica de BET (Brunauer-Emmett-Teller), obtida por absorção de nitrogênio (em um equipamento VacPrep 061 e, para a análise, utilizou-se o Gemini III 2375).

\section{Produção dos compósitos}

Foram preparadas duas diferentes formulações: aquelas produzidas sem cinzas com $100 \%$ de cimento Portland e as com cinzas com $80 \%$ de cimento e $20 \%$ de CPCA (McCarter \& Tran, 1996), além da polpa de eucalipto branqueada e das fibras de bagaço de cana-de-açúcar. A massa da polpa de eucalipto e das fibras foi de 5\% para cada uma delas de matéria seca em relação à massa do cimento. A Tabela 1 lista as dosagens de cimento e cinza, em massa, utilizadas para preparar os compósitos.

Tabela 1. Dosagens dos compósitos preparados e das matérias-primas secas (\% em massa)

\begin{tabular}{cc}
\hline Tipo de compósito & Traço em massa \\
Controle & Cimento Portland: CPCA \\
Com CPCA & $1: 0$ \\
& $0,8: 0,2$ \\
\hline
\end{tabular}

CPCA - cinza de palha de cana-de-açúcar

* Traço em massa - Teores em massa

O processo utilizado para preparação dos compósitos foi baseado no método de sucção a pressão negativa e prensagem para retirada do excesso de água (Tonoli et al., 2007). O procedimento experimental para a moldagem seguiu a sequência apresentada na Figura 1 e descrita a seguir:

A. Em um agitador de polpas com $1600 \mathrm{~mL}$ de água destilada, foram dispersas as polpas celulósicas em agitação mecânica a 3000 RPM, durante 5 min, para auxiliar na desagregação das fibras antes de se colocá-las junto ao cimento.

B. Após a desagregação a polpa foi levada a um misturador com o cimento Portland introduzindo-se, a seguir, as cinzas (quando utilizadas), as fibras e as partículas de bagaço; aplicouse agitação por 4 min na rotação de 1000 RPM.

C. A suspensão foi colocada em uma caixa de moldagem sendo aplicado o vácuo para drenar o excesso de água.

D. Realizou-se o adensamento manual até a obtenção de uma superfície sólida.


Figura 1. Sequência do processo de moldagem do compósito pelo método de sucção à pressão negativa e prensagem

E. Os compósitos foram pressionados a 3,2 $\mathrm{MPa}$, durante $5 \mathrm{~min}$

F. Após a prensagem as placas foram pesadas, identificadas e postas em sacos plásticos selados em temperatura ambiente, durante 2 dias; após este procedimento as placas foram imersas em água, durante 26 dias, para completar cura úmida.

Após os 28 dias de cura úmida as placas com dimensões iniciais de $160 \times 160 \times 6 \mathrm{~mm}$ foram cortadas em serra de disco, tendo sido produzidos quatro corpos de prova com dimensões nominais de 160 × 40 × 6 mm. Para cada formulação foram produzidas quatro placas totalizando dezesseis corpos de prova; os corpos de prova permaneceram imersos em água durante 24 $\mathrm{h}$, na condição saturada antes de serem submetidos aos ensaios físicos e mecânicos.

Uma parte dos corpos de prova foi utilizada para o estudo da durabilidade dos compósitos após a cura úmida, durante 28 dias, por meio do ensaio de envelhecimento acelerado com ciclos de imersão e secagem. Para tanto, os corpos de prova foram colocados em uma estufa banho semiautomática Marconi, modelo MA035, sendo que os ciclos foram compostos das seguintes etapas: imersão completa em água em temperatura ambiente durante $170 \mathrm{~min}$ com intervalo de $10 \mathrm{~min}$ com exposição ao ar em temperatura ambiente, aquecimento até 60 $\pm 5^{\circ} \mathrm{C}$ por igual período de $170 \mathrm{~min}$ e novo intervalo de 10 min, completando um período total de $6 \mathrm{~h}$. Este procedimento foi repetido até se completar o número desejado de 100 ciclos (Tonoli et al., 2007).

\section{Caracterização dos compósitos}

A caracterização física foi realizada para os compósitos que foram submetidos à cura úmida durante 28 dias e ao envelhecimento acelerado de 100 ciclos. Para cada mistura foram utilizados seis corpos de prova (repetições). No início foram registrados seus valores de massa saturada imersa $\left(\mathrm{M}_{\mathrm{i}}\right) \mathrm{e}$ suas massas saturadas em água com superfície seca $\left(\mathrm{M}_{\mathrm{sss}}\right)$; em seguida, os corpos de prova foram levados à estufa a $105^{\circ} \mathrm{C}$ e após $24 \mathrm{~h}$ se obtiveram os valores de massa seca das pastas $\left(\mathrm{M}_{\text {seca }}\right)$. Com os dados de massa seca $\left(\mathrm{M}_{\text {seca }}\right)$, massa saturada imersa $\left(M_{i}\right)$ e massa saturada com superfície seca $\left(M_{s s s}\right)$ foram calculadas a absorção de água (AA), expressa em \% em massa, 
a porosidade aparente (PA), expressa em \% em volume e a massa específica aparente (MAP), em $\mathrm{g} \mathrm{cm}^{-3}$, dos compósitos, com base nas Eqs. 1, 2 e 3, respectivamente.

$$
\begin{gathered}
\mathrm{AA}=\left(\frac{\mathrm{M}_{\text {sss }}-\mathrm{M}_{\text {seca }}}{\mathrm{M}_{\text {seca }}}\right) \cdot 100 \\
\mathrm{PA}=\left(\frac{\mathrm{M}_{\mathrm{sss}}-\mathrm{M}_{\text {seca }}}{\mathrm{M}_{\mathrm{sss}}-\mathrm{M}_{\mathrm{i}}}\right) \cdot 100 \\
\mathrm{MAP}=\left(\frac{\mathrm{M}_{\text {seca }}}{\mathrm{M}_{\mathrm{sss}}-\mathrm{M}_{\mathrm{i}}}\right)
\end{gathered}
$$

A caracterização mecânica dos compósitos também foi realizada para aqueles que foram submetidos à cura úmida durante 28 dias ou ao envelhecimento acelerado (100 ciclos). Para isto, foi conduzido o ensaio mecânico de tração na flexão com quatro apoios em uma máquina universal de ensaios EMIC, modelo DL-30000, equipada com uma célula de carga de $1 \mathrm{kN}$. Os vãos entre apoios superiores e inferiores foram de, respectivamente, 45 e $135 \mathrm{~mm}$. A velocidade do atuador foi de $1,5 \mathrm{~mm} \mathrm{~min}^{-1}$; a deflexão durante o teste foi coletada por meio de um defletômetro posicionado no meio do vão, na face inferior do corpo de prova. Foram utilizados dez corpos de prova (repetições) para cada mistura. Com base nos resultados do ensaio de flexão foram determinados os valores de módulo de ruptura (MOR), módulo elástico (MOE), limite de proporcionalidade (LOP) e energia específica (EE) com base nas Eqs. 4, 5, 6 e 7, respectivamente.

$$
\begin{gathered}
\text { MOR }=\frac{P_{\text {max }} \cdot L_{v}}{b \cdot h^{2}} \\
\text { MOE }=\frac{276 \cdot \mathrm{L}_{\mathrm{v}}^{3}}{1296 \cdot \mathrm{b} \cdot \mathrm{h}^{3}} \cdot \mathrm{m} \\
\mathrm{LOP}=\frac{\mathrm{P}_{\text {lop }} \cdot \mathrm{L}_{\mathrm{v}}}{\mathrm{b} \cdot \mathrm{h}^{2}} \\
\mathrm{EE}=\frac{\mathrm{EA}}{\mathrm{b} \cdot \mathrm{h}}
\end{gathered}
$$

sendo:

$\mathrm{P}_{\max }$ - força máxima aplicada ao corpo de prova

$\mathrm{L}_{\mathrm{v}} \quad$ - distância entre apoios inferiores $(135 \mathrm{~mm})$

b - largura do corpo de prova

h - espessura do corpo de prova m - tangente do ângulo de inclinação da curva força versus deflexão durante a deformação elástica

$\mathrm{P}_{\text {lop }}$ - força no limite da parte linear da curva força versus deflexão

EA - energia absorvida durante o teste de flexão, obtida pela integral da área sob a curva força versus deflexão até o ponto que corresponde à redução da capacidade da força para $30 \%$ da força máxima atingida

Os dados foram analisados por meio de um software estatístico Statgraphics Plus for Windows, versão Centurion, submetidos à análise multifatorial de variância (ANOVA) para avaliar o efeito do fator: formulações que foram produzidas (compósito controle e compósito com cinza), para os dois tipos de condição, curados aos 28 dias e envelhecidos após 100 ciclos. A comparação das médias foi feita por meio do teste de Tukey a nível de 0,05 de significância estatística.

\section{Resultados E Discussão}

As composições químicas do CPV-ARI e da CPCA estão apresentadas na Tabela 2. As cinzas atendem ao preconizado pela norma ASTM (2012) segundo a qual, para um material ser considerado pozolânico, a somatória dos óxidos $\mathrm{SiO}_{2}$, $\mathrm{Al}_{2} \mathrm{O}_{3}$ e $\mathrm{Fe}_{2} \mathrm{O}_{3}$ deve ser de, no mínimo, 50\%, e os teores de $\mathrm{SO}_{3}$ e de perda ao fogo devem ser de, no máximo, 5 e $6 \%$, respectivamente porém, segundo esta norma, o teor máximo de álcalis disponíveis em $\mathrm{Na}_{2} \mathrm{O}$ deve ser de $1,5 \%$, pois na presença desses álcalis uma pozolana pode causar problemas decorrentes da reação álcali-agregado na matriz cimentícia causando sua desintegração e a CPCA em uso apresentou o teor de 4,62\%; apesar disto, Mehta (1992) relatou a utilização de cinzas de casca de arroz como pozolana com teor de 0,05 de $\mathrm{K}_{2} \mathrm{O}$.

A difração de raios X (Figura 2) mostra as fases cristalinas presentes nas cinzas. Esta cristalização pode ser atribuída ao tempo de permanência das cinzas na mufla que foi de $14 \mathrm{~h}$, em razão da inércia térmica do forno ao ser desligado. Além do mais, quanto maior a temperatura máxima de queima do material e quanto maior o tempo de permanência do material sob altas temperaturas, maior também será sua cristalinização (Frías et al., 2007; Villar-Cociña et al., 2008; Cordeiro et al., 2009).

Além das fases cristalinas encontradas na CPCA, existe um halo de amorficidade com ângulo de Bragg (20) entre 20 e $30^{\circ}$. Materiais vítreos apresentam um halo centrado com $2 \theta$ em torno de $22^{\circ}$ e a este halo podem estar associados e sobrepostos

\begin{tabular}{|c|c|c|c|c|c|c|c|c|c|c|c|c|}
\hline \multirow{2}{*}{ Amostras } & $\mathrm{SiO}_{2}$ & $\mathrm{Al}_{2} \mathrm{O}_{3}$ & $\mathrm{~K}_{2} \mathrm{O}$ & $\mathrm{Fe}_{2} \mathrm{O}_{3}$ & $\mathrm{CaO}$ & $\mathrm{SO}_{3}$ & $\mathrm{MgO}$ & $\mathrm{P}_{2} \mathrm{O}_{5}$ & $\mathrm{TiO}_{2}$ & $\mathrm{Cl}$ & $\mathrm{MnO}$ & $\mathrm{PF}^{*}$ \\
\hline & \multicolumn{12}{|c|}{$(\%)$} \\
\hline CPCA & 61,0 & 9,2 & 7,0 & 5,0 & 4,4 & 3,9 & 2,8 & 2,3 & 1,3 & 0,6 & 0,3 & 2,1 \\
\hline CPV-ARI & 18,1 & 4,2 & 1,0 & 2,7 & 61,5 & 6,0 & 2,2 & 0,2 & 0,3 & - & - & 2,9 \\
\hline
\end{tabular}
picos de formas cristalinas da sílica (Silva, 2007). Deste modo, o halo de amorficidade é um indicativo de reatividade das cinzas.

A Figura 3 apresenta as curvas das distribuições granulométricas da CPCA e do CPV-ARI. Observa-se que

Tabela 2. Composição química de óxidos da cinza de palha de cana-de-açúcar (CPCA) e do cimento Portland de alta resistência inicial (CPV-ARI)

CPCA - cinza de palha de cana-de-açúcar; CPV-ARI - cimento Portland de alta resistência inicial

* PF - Perda ao fogo, realizada a $1000^{\circ} \mathrm{C}$. (Análise realizada no Laboratório de Caracterização Tecnológica (LCT), do Departamento de Engenharia de Minas e Petróleo da Escola Politécnica da Universidade de São Paulo) 


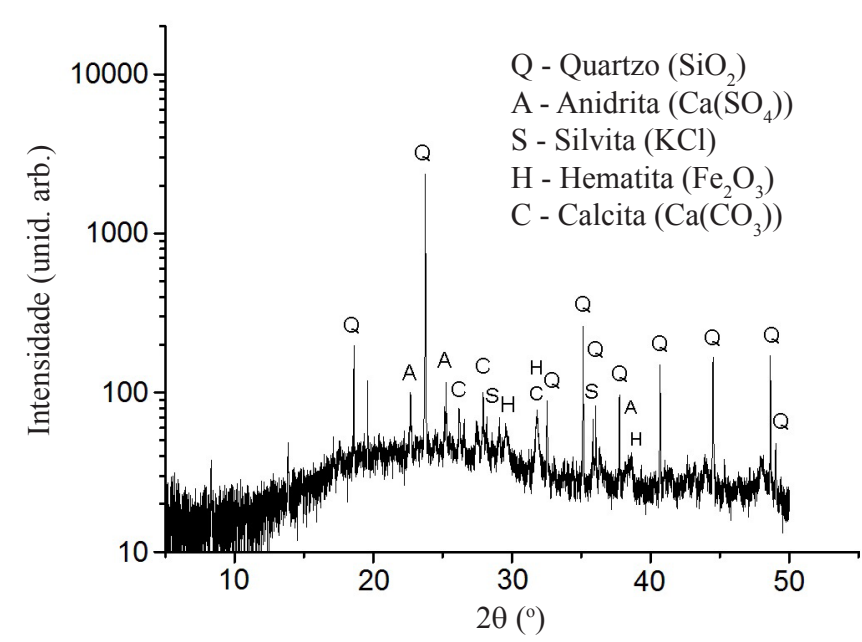

Análise realizada no Laboratório Nacional de Luz Sincrotron

Figura 2. Difratograma da cinza de palha da cana-deaçúcar (CPCA)

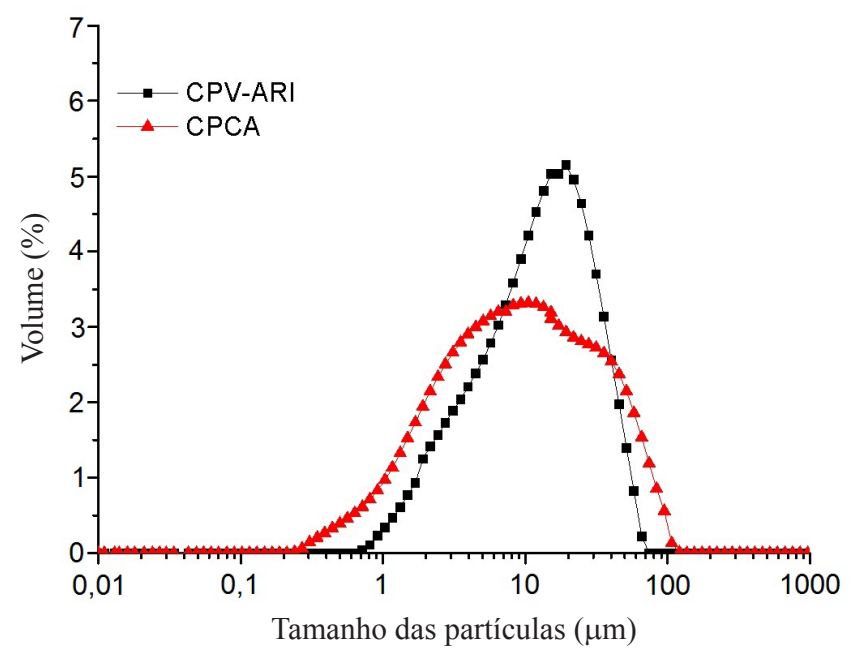

Análise realizada no LCT do Departamento de Engenharia de Minas e Petróleo da Escola Politécnica da Universidade de São Paulo

Figura 3. Distribuições granulométricas discretas da cinza de palha de cana-de-açúcar (CPCA) após a moagem e do cimento Portland de alta resistência inicial (CPV-ARI)

existe similaridade das distribuições granulométricas, discretas das cinzas após a moagem e do cimento Portland CPV-ARI.

A avaliação da superfície específica é um parâmetro importante que deve ser considerado ao se avaliar as características físicas de cinzas. $\mathrm{O}$ aumento da área superficial de um material geralmente aumenta a taxa de reação química.

Segundo Oliveira et al. (2000), a reatividade entre as partículas também está relacionada com a dispersão e o empacotamento, tal como com os teores de cada matériaprima e com sua composição química. A Tabela 3 apresenta a superfície específica da CPCA e do CPV-ARI; observa-se que

Tabela 3. Superfície específica da cinza de palha de cana-de-açúcar (CPCA) e do cimento Portland de alta resistência inicial (CPV-ARI)

\begin{tabular}{cc}
\hline Amostras & Área de superície específica $\left(\mathbf{m}^{\mathbf{2}} \mathbf{g}^{-1}\right)$ \\
CPCA & 12,20 \\
CPV-ARI & 0,91 \\
\hline
\end{tabular}

a área de superfície específica das cinzas é elevada comparada com a do cimento, em razão da sua alta porosidade.

A caracterização física dos compósitos para as três variáveis avaliadas (absorção de água, porosidade aparente e massa específica aparente, após 28 dias de cura úmida), mostrou que não houve diferença estatisticamente significativa a nível de 95\% de probabilidade pelo teste de Tukey, entre os compósitos: controle e com cinza (cim + CPCA), conforme indicado na Tabela 4; este fato indica, com relação às características físicas, que as cinzas não alteram significativamente o comportamento físico dos compósitos, aos 28 dias de cura úmida.

Tabela 4. Valores médios e os desvios-padrão das propriedades físicas dos compósitos: controle e com cinzas, aos 28 dias

\begin{tabular}{|c|c|c|c|}
\hline \multirow[t]{2}{*}{ Compósitos } & $\begin{array}{c}\text { Absorçãa } \\
\text { de água }\end{array}$ & $\begin{array}{l}\text { Porosidade } \\
\text { aparente }\end{array}$ & \multirow{2}{*}{$\begin{array}{l}\text { Massa específica } \\
\text { aparente }\left(\mathrm{g} \mathrm{cm}^{-3}\right)\end{array}$} \\
\hline & \multicolumn{2}{|c|}{$(\%)$} & \\
\hline Controle & $29,3 \pm 1,10 a$ & $41,3 \pm 1,0 a$ & $1,41 \pm 0,03 a$ \\
\hline $\mathrm{Cim}+\mathrm{CPCA}$ & $30,3 \pm 0,85 a$ & $42,1 \pm 0,8 \mathrm{a}$ & $1,39 \pm 0,02 \mathrm{a}$ \\
\hline
\end{tabular}

A Figura 4 apresenta as curvas típicas de tensão versus flecha específica dos compósitos aos 28 dias. O compósito cim + CPCA apresentou os maiores valores de tensão máxima e de flecha específica. Nota-se também, pela inclinação das curvas na região elástica, que os compósitos apresentaram rigidez similar.

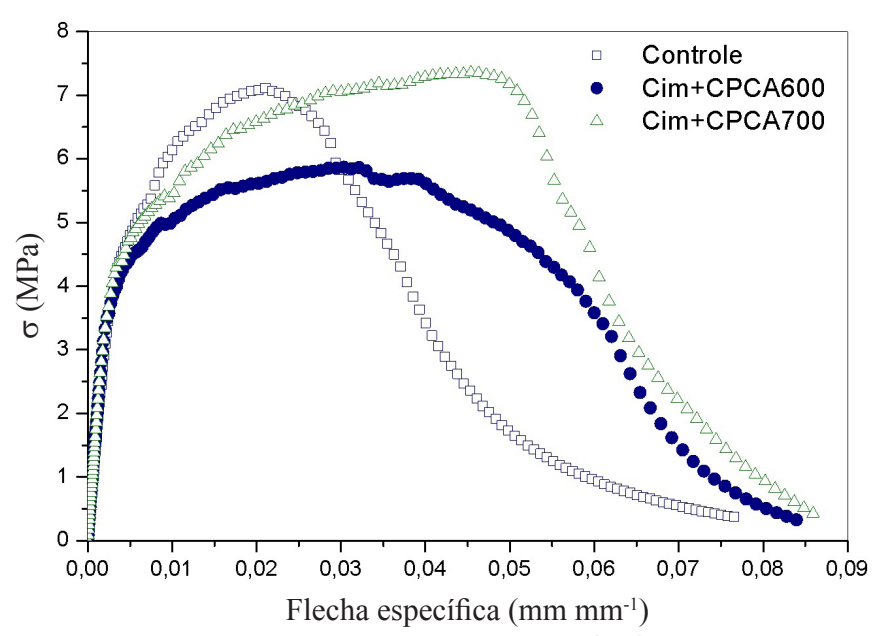

Figura 4. Curvas típicas tensão x flecha específica dos compósitos aos 28 dias de cura

Na Tabela 5 estão apresentados os valores das médias e os erros-padrão do módulo de ruptura (MOR), do limite de proporcionalidade (LOP), da razão $M O R / L O P$, da energia específica (EE) e do módulo de elasticidade (MOE) para os compósitos controle e cim + CPCA, ensaiados após 28 dias de cura úmida.

O módulo de ruptura pode ser relacionado com um provável reforço da fibra na matriz cimentícia. Quanto ao LOP, indica a tensão máxima atingida na região elástica antes da ocorrência da primeira fissuração do compósito, observando-se que houve diferença estatisticamente significativa entre os valores médios dos compósitos. 
Tabela 5. Valores médios e desvios-padrão das propriedades mecânicas no ensaio de flexão dos compósitos controle e com cinzas para 28 dias de cura

\begin{tabular}{ccccccc}
\hline Compósitos & MOR & LOP & MOR/LOP & EE & MOE \\
\cline { 2 - 3 } Controle & & (MPa) & & & & (GPa) \\
Cim + CPCA & $7,13 \pm 1,5 \mathrm{a}$ & $2,92 \pm 0,6 \mathrm{a}$ & $2,43 \pm 0,68 \mathrm{~b}$ & $2,14 \pm 0,8 \mathrm{a}$ & $6,58 \pm 0,63 \mathrm{a}$ \\
\hline
\end{tabular}

CPCA - cinza de palha de cana-de-açúcar; MOR - módulo de ruptura; LOP - limite de proporcionalidade; EE - energia específica; MOE - módulo de elasticidade

* Colunas com médias seguidas de letras diferentes apresentam diferença estatisticamente significativa entre si pelo Teste de Tukey a 0,05 de probabilidade

A razão MOR/LOP dos compósitos ensaiados aos 28 dias de cura também está apresentada na Tabela 5 . O compósito cim + CPCA apresentou um valor mais elevado de MOR/LOP e, além disto, houve diferença estatisticamente significativa em relação ao compósito controle. Esta razão indica que as fibras vegetais contribuíram para aumentar a resistência à tração na flexão, ou seja, para os compósitos cim + CPCA observou-se que as fibras contribuíram de forma mais efetiva no aumento da resistência. Este comportamento das fibras pode ser decorrente do fato de que, ao se incorporar as cinzas neste compósito, as mesmas podem ter consumido hidróxido de cálcio e, contrapartida, diminuído a alcalinidade da matriz colaborando, então, para a proteção das fibras neste compósito.

Com relação à EE, que é uma propriedade que permite efetuar-se a avaliação da resistência ao impacto e da contribuição das fibras para a tenacidade do compósito, observou-se que os compósitos cim + CPCA apresentaram valor aproximadamente $50 \%$ acima do valor do compósito controle cujo resultado corrobora com o da razão MOR/LOP mais elevada para este compósito. Deste modo, a CPCA colaborou para a proteção devido à redução da alcalinidade e melhoria na aderência das fibras vegetais à matriz cimentícea. No entanto, para a variável MOE não houve diferença estatisticamente significativa para os compósitos produzidos neste trabalho.

$\mathrm{Na}$ Tabela 6 se encontram os valores das médias e os desvios-padrão da caracterização física dos compósitos avaliados após serem submetidos a 100 ciclos de imersão e secagem. Para as três variáveis consideradas: absorção de água, porosidade aparente e massa específica aparente, houve diferença estatisticamente significativa a nível de 0,05 de probabilidade pelo teste de Tukey, entre os compósitos.

Tabela 6. Valores médios e desvios-padrão das propriedades físicas dos compósitos: controle e com cinzas, após 100 ciclos de imersão e secagem

\begin{tabular}{lccc}
\hline \multirow{2}{*}{ Compósitos } & \multicolumn{1}{c}{$\begin{array}{c}\text { Absorçãa } \\
\text { de água }\end{array}$} & $\begin{array}{c}\text { Porosidade } \\
\text { aparente }\end{array}$ & $\begin{array}{c}\text { Massa específica } \\
\text { aparente }\left(\mathbf{g ~ c m}^{-3}\right)\end{array}$ \\
\cline { 2 - 3 } Controle & \multicolumn{2}{c}{$\mathbf{( \% )}$} & \\
CIM/CPCA & $23,3 \pm 1,4 \mathrm{a}$ & $34,1 \pm 1,5 \mathrm{a}$ & $1,47 \pm 0,03 \mathrm{a}$ \\
& $25,3 \pm 0,9 \mathrm{~b}$ & $35,5 \pm 0,7 \mathrm{a}$ & $1,41 \pm 0,03 \mathrm{~b}$ \\
\hline
\end{tabular}

CPAC - cinza de palha de cana-de-açúcar

* Colunas com médias seguidas de letras diferentes apresentam diferença estatisticamente significativa entre si pelo Teste de Tukey a 0,05 de probabilidade
O valor médio da porosidade aparente do compósito cim + CPCA não apresentou diferença estatisticamente significativa quando foi comparado com o do controle; mesmo assim, este compósito apresentou elevado valor médio de absorção de água; o compósito controle apresentou um valor mais elevado de massa específica aparente tendo apresentado diferença estatisticamente significativa com relação ao compósito com cinzas. Comparando os valores de absorção de água e de porosidade aparente obtidos após 28 dias de cura com aqueles referentes aos compósitos após serem submetidos ao envelhecimento acelerado de 100 ciclos de imersão e a secagem, observou-se que ambas as propriedades físicas apresentaram diminuição aproximada de $15 \%$, que pode ser atribuída ao fenômeno de colmatação dos poros decorrente da formação de novos produtos de hidratação a partir das reações pozolânicas desenvolvidas.

As curvas típicas de tensão versus flecha específica estão apresentadas na Figura 5. O compósito controle apresentou comportamento mecânico frágil, após ser submetido ao envelhecimento acelerado por imersão e secagem de 100 ciclos.

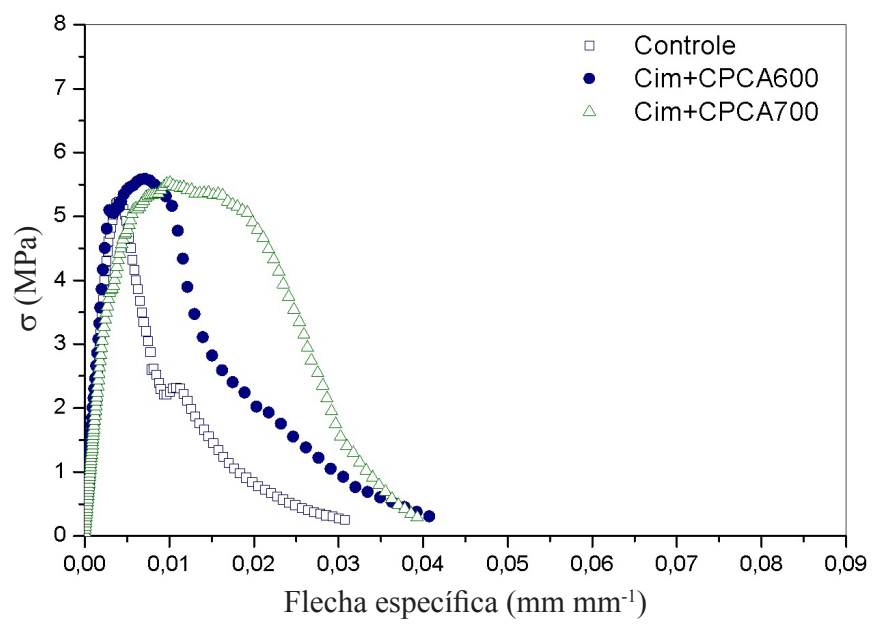

Figura 5. Curvas típicas tensão $(\sigma)$ x flecha específica dos compósitos após 100 ciclos de imersão e secagem

Na Tabela 7 estão apresentados os valores das médias e os desvios-padrão da caracterização mecânica e a razão MOR/ LOP dos compósitos, após serem submetidos a 100 ciclos de envelhecimento acelerado por imersão e secagem.

Tabela 7. Valores médios e desvios-padrão das propriedades mecânicas dos compósitos avaliados após 100 ciclos de envelhecimento de imersão e secagem

\begin{tabular}{|c|c|c|c|c|c|}
\hline Compósitos & MOR & LOP & MOR/LOP & EE & $\begin{array}{l}\text { MOE } \\
\text { (GPa) }\end{array}$ \\
\hline $\begin{array}{c}\text { Controle } \\
\text { Cim + CPCA }\end{array}$ & $\begin{array}{l}5,00 \pm 0,6 a \\
5,48 \pm 0,6 a\end{array}$ & $\begin{array}{l}3,75 \pm 0,9 a \\
2,98 \pm 0,6 b\end{array}$ & $\begin{array}{r}1,33 \pm 0,27 b \\
1,84 \pm 0,60 a\end{array}$ & $\begin{array}{l}0,32 \pm 0,8 b \\
0,86 \pm 0,8 \mathrm{a}\end{array}$ & $\begin{array}{l}6,99 \pm 0,89 a \\
6,02 \pm 0,78 b\end{array}$ \\
\hline
\end{tabular}

CPCA - cinza de palha de cana-de-açúcar; MOR - módulo de ruptura; LOP - limite de proporcionalidade; EE - energia específica; MOE - módulo de elasticidade 
Após os ciclos observou-se diminuição nos valores de MOR, conforme observado na Figura 6. Este comportamento era esperado, visto que o material passou por um ensaio agressivo de envelhecimento acelerado que estimulou o ataque da água alcalina nos poros da fibra celulósica. Para esta variável não houve diferença estatisticamente significativa entre os compósitos, a nível de 0,05 de probabilidade, pelo teste de Tukey.

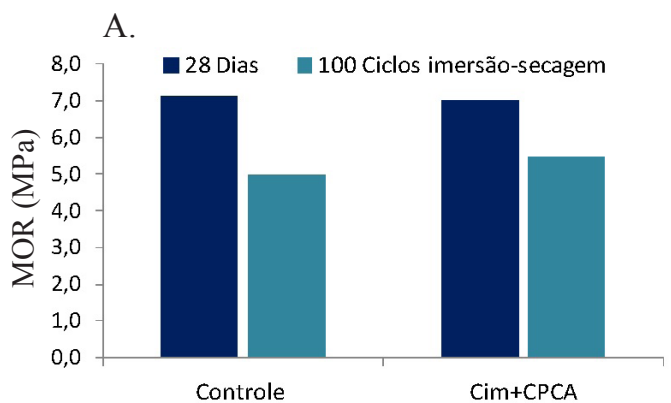

B.

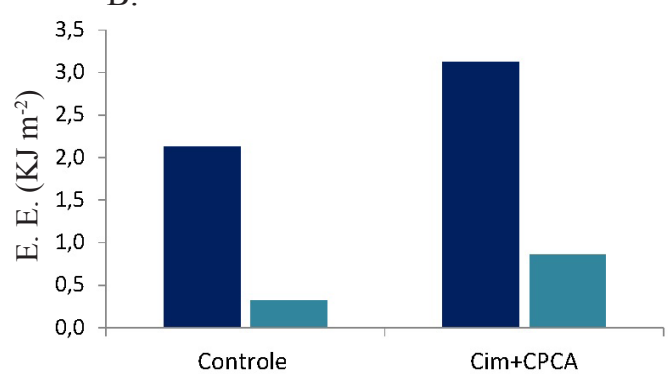

C.

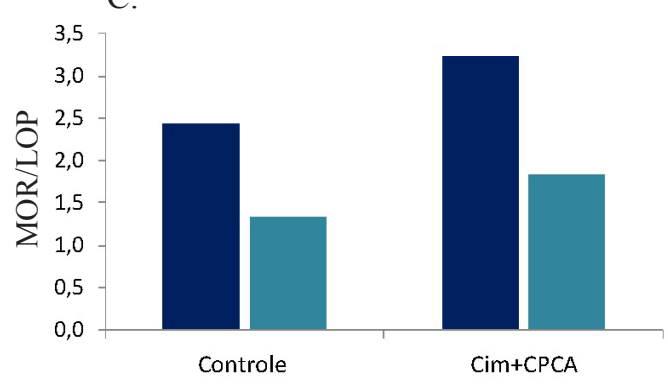

CPAC - cinza de palha de cana-de-açúcar

Figura 6. Médias de módulo de ruptura - MOR (A), energia específica - EE (B) e MOR/LOP, dos compósitos (C): controle e cim + CPCA, após 28 dias de cura úmida e 100 ciclos de imersão e secagem

Para a variável MOE, constatou-se diferença estatisticamente significativa entre os compósitos, sendo que o controle apresentou o comportamento mecânico mais rígido dentre eles, com valor médio de MOE superior, portanto, ao dos compósitos com cinzas.

O compósito com cinzas, após sofrer 100 ciclos de envelhecimento, apresentou maior razão MOR/LOP em relação compósito controle. Apesar da queda apresentada da razão MOR/LOP dos compósitos avaliados após 100 ciclos de envelhecimento em relação aos compósitos avaliados após a cura úmida de 28 dias (Figura 6), observou-se novamente que o desempenho mecânico das fibras no compósito com cinzas foi superior ao do compósito controle.

Este resultado corrobora com as análises dos valores médios da EE para esses compósitos, embora se tenha observado uma diminuição considerável dos valores de EE em relação aos valores obtidos após 28 dias de cura, o compósito com CPCA apresenta valores de EE superiores aos do compósito controle fenômeno este passível de ser observado também por meio da área sob a curva apresentada na Figura 5 na qual estão apresentados os dados de tensão versus flecha específica e a área sob a curva do compósito cim + CPCA é superior à área do compósito controle.

Tais resultados físicos e mecânicos dos compósitos cim + CPCA, indicam refinamento dos poros da matriz e da zona de transição fibra-matriz além da maior conservação das fibras celulósicas no interior da matriz menos agressiva, em razão da adição pozolânica.

Concluiu-se, com isto, que a aplicação de cinzas de palha de cana-de-açúcar com queima controlada a $700{ }^{\circ} \mathrm{C}$ se torna uma opção para o aproveitamento desse resíduo agroindustrial como adição mineral ao cimento propondo-se, desta forma, uma nova aplicação para o referido resíduo cuja produção deverá ser incrementada com o processo de colheita mecanizada no estado de São Paulo.

\section{CONClusÕES}

1. As caracterizações físicas e químicas da cinza de palha de cana-de-açúcar (CPCA) mostraram que parte do material é amorfo que apresenta elevada porosidade interna e que a somatória dos principais compostos químicos mostrou-se acima de $75 \%$ podendo ser considerada um material pozolânico.

2. Após a moagem as cinzas apresentaram distribuição de partículas muito finas e elevadas nano e microporosidade.

3. Os compósitos produzidos com a cinza de palha de canade-açúcar (CPCA) aos 28 dias de cura úmida, apresentaram características físicas, tal como valores médios de módulo de ruptura na flexão similares aos do controle.

4. Os compósitos avaliados após o envelhecimento acelerado por meio de 100 ciclos de imersão e secagem com cinza, apresentaram diferença estatisticamente significativa em relação às propriedades de absorção de água e massa específica aparente. Esses compósitos apresentaram maior razão MOR/LOP e maior valor médio de energia específica em relação ao controle.

\section{Agradecimentos}

Ao $\mathrm{CNPq}$, pelo apoio financeiro em prol da realização da pesquisa e pelas Bolsas de Doutorado e Produtividade em pesquisa aos autores; ao CTC - Centro de Tecnologia Canavieira, pelo fornecimento da palha de cana-de-açúcar; à Abengoa Bioenergia, pelo fornecimento do bagaço de canade-açúcar; ao Laboratório Nacional de Luz Sincrotron e ao Laboratório de Construções e Ambiência da FZEA/USP, nos quais foram realizados o ensaio de difração de raios $\mathrm{X}$ da cinza e os ensaios físicos e mecânicos dos compósitos.

\section{Literatura Citada}

Agopyan, V.; Savastano Jr., H. Compósitos cimentícios reforçados com fibras vegetais e suas aplicações. In: Tecnologia e materiais alternativos de construção. Freire, W. J.; Beraldo, A. L.(coord.). Campinas: UNICAMP, 2003. p.121-142. 
ASTM - American Society for Testing and Materials: ASTM C 618 - Standard specification for coal fly ash and raw or calcined natural pozzolan for use in concrete. West Conshohocken: ASTM, 2012. 3p.

Bui, D. D.; Hu, J.; Stroeven, P. Particle size effect on the strength of rice husk ash blended gap-graded Portland cement concrete. Cement \& Concrete Composites, v.7, p.357-366, 2005.

Cordeiro, G. C.; Toledo Filho, R. D.; Fairbairn, E. M. R. Effect of calcination temperature on the pozzolanic activity of sugar cane bagasse ash. Construction and Building Materials, v.23, p.3301-3303, 2009.

Dwivedi, V. N.; Singh, N. P.; Dasa, S. S.; Singh, N. B. A new pozzolanic material for cement industry: Bamboo leaf ash. International Journal of Physical Sciences, v.1, p.106-111, 2006.

Frías, M.; Rojas, M.I. S. de. Influence of metastable hydrated phases on the pore size distribution and degree of hydration of MK-blended cements cured at $60{ }^{\circ} \mathrm{C}$. Cement and Concrete Research, v.35, p.1292-1298, 2005.

Frías, M.; Villar-Cociña, E.; Valencia-Morales, E. Characterization of sugar cane straw waste as pozzolanic material for construction: Calcining temperature and kinetic parameters. Waste Management, v.27, p.533-538, 2007.

Frías, M.; Villar, E.; Savastano Jr., H. Brazilian sugar cane bagasse ashes from the cogeneration industry as active pozzolans for cement manufacture. Cement \& Concrete Composites, v. 33, p. 490-496, 2011.

Ganesan, K.; Rajagopal, K.; Thangavel, K. Evaluation of bagasse ash as supplementary cementitious material. Cement \& Concrete Composite, v.29, p.515-524, 2007.

Guzmán, A., Gutiérrez, C., Amigó, V., Mejíade Gutiérrez, R., Delvasto, S. Valoración puzolánica de la hoja de la caña de azúcar. Materiales de Construcción, v.61, p.213-225, 2011.

Isaia, G. C.; Gastaldini, A.L.G.; Moraes, R. Physical and pozzolanic action of mineral additions on the mechanical strength of high-performance concrete. Cement e Concrete Composites, v.25, p.69-76, 2003.

Lima, P. R. L.; Toledo Filho, R.D. Uso de metacaulinita para incremento da durabilidade de compósitos à base de cimento reforçados com fibras de sisal. Ambiente Construído, v.84, p.7-19, 2008.

Martirena, F.; Middendorf, B.; Day, R.L.; Gehrke, M.; Roque, P.; Martínez, L.; Betancourt, S. Rudimentary, low tech incinerators as a means to produce reactive pozzolan out of sugar cane straw. Cement and Concrete Research, v.36, p.1056-1061, 2006.

McCarter, W. J.; Tran, D. Monitoring pozzolanic activity by direct activation with calcium hydroxide. Construction and Building Materials, v.10, p.179-184, 1996.

Mehta, P. K. Rice husk ash - A unique supplementary cementing material. In: Advances in concrete technology. Malhotra, V. M. (ed.), 2.ed., Ottawa: Canmet, 1992. p.407-431.
Mishra, S. R.; Kumar, S.; Park, A.; Rho, J.; Losby, J.; Hoffmeister, B.K. Ultrasonic characterization of the curing process of PCC fly ash-cement composite. Materials Characterization, v.7, p.317-323, 2003.

Mitchell, D. R. G.; Hinczak, I.; Day, R. A. Interaction of silica fume with calcium hydroxide solutions and hydrated cement pastes. Cement and Concrete Research, v.28, p.1571-1584, 1998.

Nita, C. Utilização de pozolanas em compósitos de cimento reforçados com fibras de celulose e PVA. São Paulo: USP, 2006.112p. Dissertação Mestrado

Oliveira, I., R.; Studart, A. R.; Pileggi R. G.; Pandolfelli, V. C. Dispersão e empacotamento de partículas: Princípios e aplicações em processamento cerâmico. São Paulo: Fazendo Arte, 2000. 195p.

Payá, J.; Monzó, J.; Borrachero, M. V.; Mellado, A.; Ordonez, L. M. Determination of amorphous silica in rice husk ash by a rapid analytical method. Cement and Concrete Research, v.31, p.227-231, 2001.

Peruzzi, A. P. Estudo das alternativas da fibra de vidro sem características álcali resistente em elementos construtivos de cimento Portland. São Carlos: USP, 2007. 182p. Tese Doutorado

Sata, V.; Tangpagasit, J.; Jaturapitakkul, C.; Chindaprasirt, P. Effect of W/B ratios on pozzolanic reaction of biomass ashes in Portland cement matrix. Cement e Concrete Composites, v.34, p. 94-100, 2012.

Silva, M. G. Cimentos Portland com adições minerais. In: Materiais de construção civil e princípios de ciências e engenharia de materiais. Isaia, G. C. (ed.). São Paulo: Ibracon, 2007. p.761-793.

SNIC - Sindicato Nacional da Indústria do Cimento. Relatório anual de 2010. < http://www.snic.org.br >. 5 Jan. 2012.

Tashima, M. M.; Souza, L. C.; Akasaki, J. L.; Silva, E. J.; Melges, J. L. P.; Payá, J. J. B. Reuse of rice husk ash in building constructions. HolosEnvironment, v.11, p.81-89, 2011

Toledo Filho, R. D.; Ghavami, K.; England, G. L.; Scrivener, K. Development of vegetable fibre-mortar composites of improved durability. Cement \& Concrete Composites, v.25, p.185-196, 2003.

Tonoli, G. H. D.; Joaquim, A. P.; Arsène, M. A.; Bilba, K.; Savastano Jr., H. Performance and durability of cement based composite reinforced with refined sisal pulp. Materials and Manufacturing Process, v.22, p.149-156, 2007.

Tonoli, G. H. D.; Santos, S. F.; Rabi, J. A.; Santos, W. N.; Savastano Jr., H. Thermal performance of sisal fiber-cement roofing tiles for rural constructions. Scientia Agricola, v.68, p.1-7, 2011.

Villar-Cociña, E.; Frias, M. R.; Valencia-Morales, E. Sugar cane as pozzolanic materials: application of a mathematical model. ACI Mater Journal, v.105, p.258-264, 2008. 